\title{
Design study of integrated photonic transmitters for application in Fiber-to-the-Home networks
}

\author{
Katarzyna Ławniczuk ${ }^{* 1,2}$, Ryszard Piramidowicz ${ }^{2}$, Paweł Szczepański ${ }^{2}$, Meint Smit ${ }^{1}$ and Xaveer Leijtens ${ }^{1}$ \\ ${ }^{1}$ COBRA Research Institute, Eindhoven University of Technology, the Netherlands, \\ ${ }^{2}$ Institute of Microelectronics and Optoelectronics, Warsaw University of Technology, Poland
}

Received June 21, 2010; accepted June 28, 2010; published June 30, 2010

\begin{abstract}
Different solutions of photonic transmitters based on arrayed waveguide gratings are demonstrated. The basic concepts of realizing integrated multiwavelength lasers with optical modulators are presented and discussed in detail.
\end{abstract}

The realization of next generation optical communication networks requires integrating a large number of optical components and offering multiple services with continuously increasing bandwidth demands. Photonic integration technology may satisfy these requirements by fabricating compact, multifunctional and high-speed components. Despite active elements, like semiconductor optical amplifiers, light sources and detectors, one of the key components of integrated photonic systems is the arrayed waveguide grating (AWG). AWG can be made in almost any waveguide technology and is suitable for integration with other optical components [1]-[2]. The main applications of AWGs are multiplexers and de-multiplexers in WDM systems, add-drop filters, optical cross-connects, channel selectors, wavelength routers, wavelength-selective switches, multiwavelength receivers and transmitters [1] [7]. Multiwavelength sources of simultaneous emission based on AWGs can be integrated with high-speed modulators. This combination yields an increase in the bandwidth and transmission capacity of networks which is expected to play a crucial role in the development of Fiber-to-the-Home systems.

A schematic view of the AWG structure is presented in Fig. 1. The operational principle of the structure is as follows. First, a number of wavelengths propagate through a transmitter waveguide. After that they enter the first free propagation region (FPR) where they are no longer laterally confined. The beam of light starts to diverge and at the input aperture it is coupled into a waveguide array of AWG. The length of array waveguides is chosen so that the optical path length difference between adjacent waveguides equals an integer multiple of the central wavelength. The light propagates through the arms of AWG and reaches output aperture. In

\footnotetext{
*E-mail: k.lawniczuk@tue.nl; k.lawniczuk@elka.pw.edu.pl
}

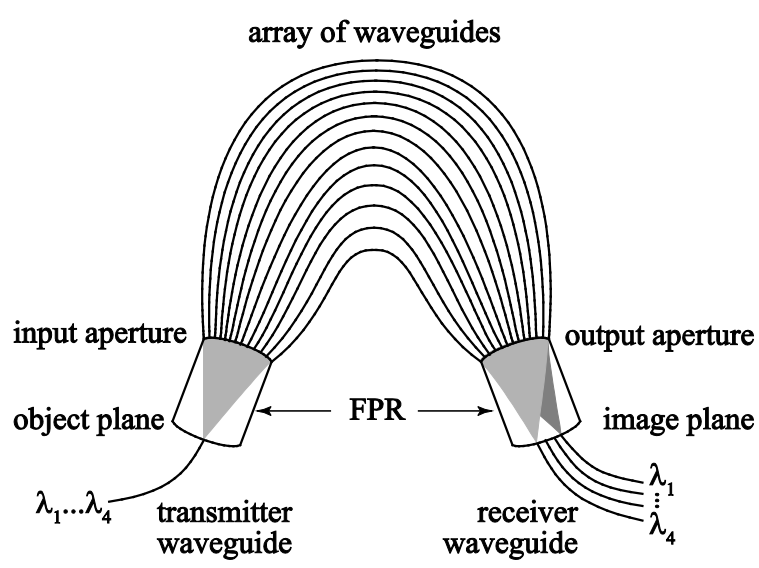

Fig. 1. Schematic of arrayed waveguide grating [1].

the second FPR light becomes convergent and constructively interferes in the image plane. The beam of the central wavelength is projected at the center of the image plane. Because of dispersion properties of the AWG the phases of the beams of other wavelengths change. This results in tilting and shifting of the focal point of the beams along the image plane. By placing receiver waveguides at proper positions in the image plane, different wavelength channels can be spatially separated.

AWG based lasers can be realized in many configurations while the most common one is presented in Fig. 2. It consists of an array of semiconductor optical amplifiers (SOAs) integrated with an AWG. The cleaved facets of the chip act as highly-reflective (HR) and partially-reflective (PR) mirrors which together with

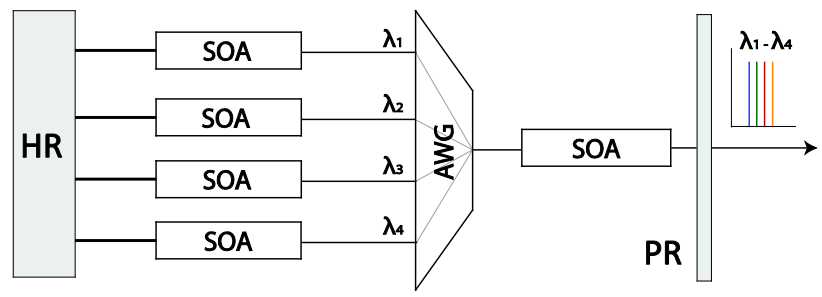

Fig. 2. Schematic of AWG based laser.

(C) 2010 Photonics Society of Poland 


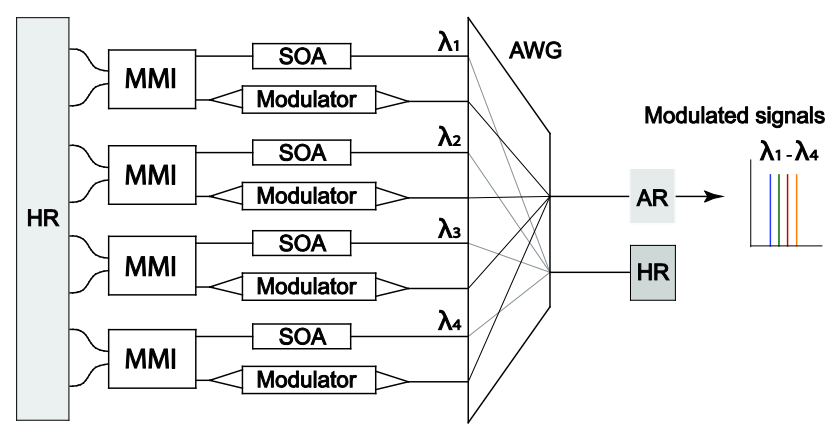

Fig. 3. Schematic of AWG laser integrated with four electro-optical modulators.

semiconductor optical amplifiers form a laser with a Fabry-Pérot cavity. The reflection and transmission properties of the cleaved facets can be chosen by applying a metal or dielectric coating. If the gain of an amplifier overcomes the propagation and facet losses, lasing is observed. Wavelength selection is determined by the AWG, which acts as an intra-cavity filter. The cavity loss is minimal for the specific wavelength corresponding to the input port of given SOA and passband of AWG. For each generated wavelength a separate SOA is dedicated. Total optical power can be increased by placing a booster amplifier between an AWG and output coupler. The lasing wavelengths of such AWG-based lasers are fully determined by the AWG passband location and channel spacing. AWG-based lasers are able to simultaneously generate several wavelengths, and have good operational stability at a given wavelength. Such devices were already demonstrated by a number of authors [3]-[4], [6]. However, to obtain a fully operational multiwavelength transmitter, the integration of a laser with radio-frequency modulators is required.

Both electro-optical Mach-Zehnder modulators (MZMs) and electro-absorption modulators (EAMs) are suitable for such designs. EAMs are widely used in optical communication to provide high-bit rate modulation (over $10 \mathrm{~Gb} / \mathrm{s}$ ). They are characterized by their small size, low driving voltage, but their saturation power is low. In addition, EAMs could be rather complex and costly in fabrication processes [8].

Mach-Zehnder structures, typically used in long-haul optical communication systems, have the advantage of wavelength independent behavior, long term stability and can handle high optical power. They may introduce frequency chirp and relaxation oscillations, and thus limit the modulation performance in high bit-rate systems [9][10].

The next paragraph will describe the design process of devices, which could simultaneously transmit multiple modulated signals.

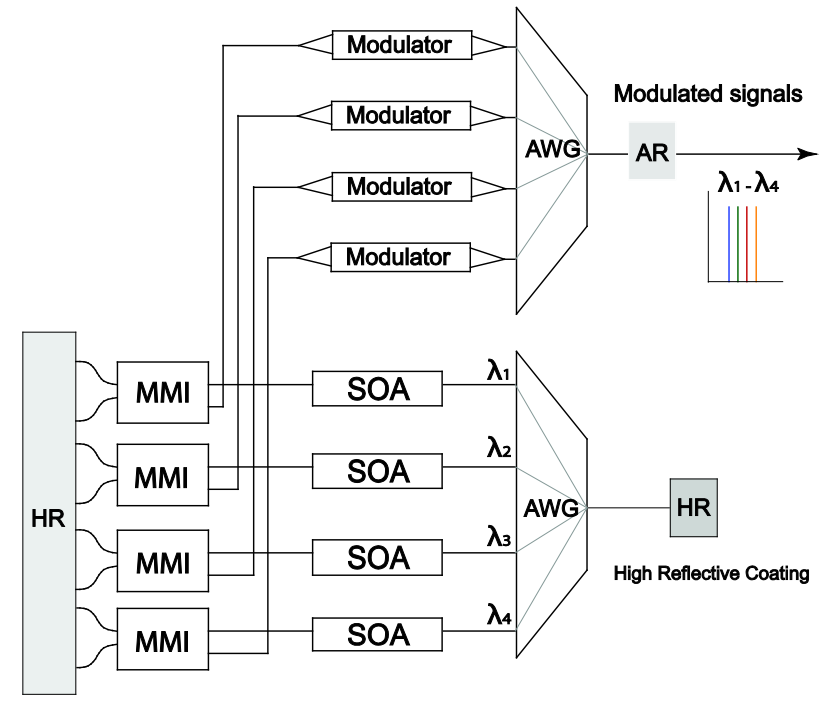

Fig. 4. Schematic of AWG laser integrated with electro-optical modulators and AWG acting as multiplexer.

AWG based multiwavelength lasers can be integrated with fast modulators in several ways. To maintain laser stability and exclude frequency chirping originating in the SOA, the modulator should be placed outside the laser cavity. The schemes of possible configurations are given in Fig. 3 and Fig. 4.

Figure 3 shows the schematic of a multiwavelength transmitter with an AWG used as an intra-cavity filter that combines modulated signals. In this configuration a larger AWG is required with a doubled number of channels that are separated by half of channel spacing. Modulators and SOAs are placed in the input waveguides of the AWG. The laser cavity is formed between the cleaved facets covered with a highly reflective coating (HR). A fraction of the generated light is coupled out from the cavity using for instance, a multimode interferometer (MMI) as a 3-dB splitter. The signals are routed to the modulators and then to the AWG, which acts as a multiplexer forwarding the modulated beam to the output waveguide. An anti-reflective coating (AR) is used to decrease reflective losses caused by the cleaved facets.

Another solution of a multiwavelength transmitter is presented in Fig. 4. Here two AWGs are integrated to form a transmitter that modulates multiple signals. The AWG drawn lower in the figure is used to form a laser cavity. The upper AWG works as a multiplexer to combine all signals into a common output. A fraction of the generated light is coupled out from the cavity using a 3-dB MMI splitter. After that, the modulation of signals takes place in the modulators (see the upper part of the figure). Finally, the light is routed to multiplexing AWG and from there to the common output waveguide. Highly 
reflective and anti-reflective coatings reduce reflective losses in the structure.

The described solutions of integrated transmitters can be improved using multiwavelength lasers with filtered feedback for wavelength locking. The designs, presented above, use an AWG as intra-cavity filter. That is why the laser cavity becomes rather long. A promising alternative is to implement on-chip reflectors that partially reflect and transmit optical signals. A scheme of a structure that takes advantage of high-contrast distributed Bragg reflector (DBR) mirrors is given in Fig. 5. Wavelength selection is achieved by feedback from the AWG placed this time outside the laser cavity. In this design the laser is formed by two Bragg gratings and the semiconductor optical amplifier in between. Locking the laser to the specific wavelength is achieved by the feedback from the AWG. The laser cavity is short and the length, and therefore the longitudinal mode-spacing, is defined with lithographic precision by the position of the DBR mirrors. When lasing occurs in such a structure, a fraction of the light leaves the cavity through a partially reflecting DBR mirror and is routed to the modulator. Finally, the beam seen as a modulated signal is directed to the output waveguide by the AWG.

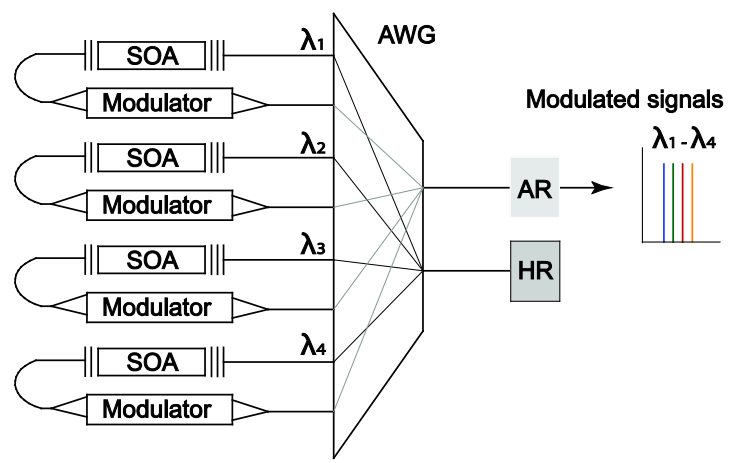

Fig. 5. Schematic of a multiwavelength source integrating AWG, SOAs, Bragg gratings and electro-optical modulators. Multi-wavelength laser uses filtered feedback for wavelength locking.

\section{Summary}

A number of concepts to realize multiwavelength lasers integrated with modulators were developed during the last few years. In this paper we discussed the most promising of them and gave a detailed account of the operating principles of the AWG based transmitters for applications in multiwavelength Fiber-to-the-Home systems. The presented designs are the subject of intensive development. At present mask layouts of the devices are being prepared and fabrication of the AWG based multiwavelength transmitters is in progress. The results will be reported in the upcoming publications.

This work is a part of the European Seventh Framework Programme, grant agreement no.: CP-TP 228839-2 EuroPIC.

\section{References}

[1] M. K. Smit, Electron. Lett. 24(7), 385 (1988).

[2] M.K. Smit, 3rd Int. conference on Optics-photonics Design and Fabrication, 51-52, Tokyo 1, 30 Oct. - 1 Nov. 2002, Optical Society of Japan, 2002.

[3] J.H. den Besten, Integration of Multiwavelength Lasers with Fast Electro-Optical Modulators, PhD Thesis, 2004.

[4] B.Docter, Deeply-Etched DBR Mirrors for Photonic Integrated Circuits and Tunable Lasers, PhD Thesis, 2009.

[5] S.C. Nicholes, M.L. Ma`sanovic', B. Jevremovic', E. Lively, L.A. Coldren, J. Lightwave Techn. 28(4) Feb. (2010).

[6] R.Broeke, A Wavelength Converter Integrated with a Discretely Tunable Laser for Wavelength Division Multiplexing Networks, $\mathrm{PhD}$ Thesis, 2003.

[7] F.M. Soares, J.H. Baek, N.K. Fontaine, C. Junesand, S. Lourdudoss, K.Y. Liou, R.A. Hamm, W. Wang, B. Patel, W.S. Hobson, J.R. Lothian, S. Vatanapradit, L.A. Gruezke, W.T. Tsang, and S.J.B. Yoo, Optical Fiber Communications Conference, Paper OThS1, 2010

[8] H. Yang, M. Chin, J. Zhou, S. Lee, W. Chen, and H. Zhu, Semicond. Sci. Technol. 23, 105011 (2008)

[9] J.H. den Besten, D. Caprioli, W. Pascher, R. van Dijk, X.J.M. Leijtens, and M.K. Smit, Symposium IEEE/LEOS Benelux Chapter, Dec. 2002.

[10] H.N. Klein, H. Chen, D. Hoffmann, S. Staroske, A.G. Steffan, K.-O. Velthaus, International Conference on Indium Phosphide and Related Materials Conference Proceedings, 171-173, 2006. 